\title{
First-Principles Methodology for Determining the Angular Momentum of Excitons
}

\author{
Xiaoning Zang ${ }^{1}$ and Udo Schwingenschlögl ${ }^{1, *}$ \\ ${ }^{1}$ Physical Sciences and Engineering Division (PSE), \\ King Abdullah University of Science and Technology \\ (KAUST), Thuwal, 23955-6900, Saudi Arabia
}

\begin{abstract}
We develop a methodology for extracting the Kohn-Sham angular momentum of excitons in realistic systems from time-dependent density functional theory. For small systems the exciton populations can be calculated analytically, which allows us to test the methodology for a 3 -arm $\mathrm{H}_{2}$ molecular ring and a pair of such rings. For larger systems the developed methodology opens for the first time a venue to determine the angular momentum of excitons by first principles calculations. A chain of twenty 3-arm $\mathrm{H}_{2}$ molecular rings and a triphenylphosphine molecule are investigated as illustrative examples. It is demonstrated that the angular momentum is conserved during the absorption of twisted light.
\end{abstract}




\section{INTRODUCTION}

As angular momentum (AM) can serve as extra degree of freedom for encoding information ${ }^{1}$, research efforts towards utilization of twisted light (light carrying AM) have been made both in classical ${ }^{2-4}$ and quantum communication ${ }^{5-15}$. A variety of setups for generating twisted light have been developed, such as spatial light modulators ${ }^{16,17}$, spiral phase plates $^{18}$, q plates ${ }^{19-22}$, homogeneous uniaxial birefringent crystals $^{23-27}$, molecular arrays ${ }^{28,29}$, and metasurfaces ${ }^{30-32}$. Experiments have demonstrated distinguishable modes of twisted light even after a transmission of $143 \mathrm{~km}^{33}$. In addition, quantum entanglement of states with $\mathrm{AM}>10000$ has been observed in Ref. 34 .

Only recently there are attempts to manipulate the AM of twisted light by excitons ${ }^{35,36}$. An exciton is a unit vector on a Bloch sphere when considered as quantum bit and can be rotated by applying laser pulses ${ }^{37-44}$ to realize operations of quantum logic gates. There exist devices for storing excitons ${ }^{45,46}$ and controlling excitons by electric fields ${ }^{47,48}$. In addition, gating architectures ${ }^{49}$, excitonic transistors ${ }^{50-52}$, exciton conveyors ${ }^{53}$, and devices for directed exciton transfer ${ }^{54,55}$ have been reported. This technical progress makes it possible to employ excitons to efficiently process and manipulate information encoded in light by bridging the gap between photonics and electronics ${ }^{56}$. An exciton that is generated by illuminating a molecule with $C_{N}(N \geq 3)$ symmetry by twisted light, for example, obtains the AM of the absorbed photon, and it turns out that a chain of such molecules can be used to create excitonic wave packets with well defined linear and angular momenta ${ }^{35,36,57}$. A succession of absorption events generates excitonic wave packets carrying a range of AM. The fact that the modified AM can be transferred back to a photon by emission implies that excitons are able to mediate a change in the AM of twisted light.

In order to examine excitons in real molecules, such as triphenylphosphine, hexaphenylbenzene, and coronene, we develop in the present work a methodology for extracting the Kohn-Sham (KS) AM from calculations within time-dependent density functional theory (TDDFT). This first-principles methodology is first tested for a 3-arm $\mathrm{H}_{2}$ molecular ring and a pair of such rings, because the populations of excitons with different AM can be calculated analytically for both these systems. Afterwards, we apply it to a chain of twenty 3-arm $\mathrm{H}_{2}$ molecular rings as well as to a triphenylphosphine molecule. 


\section{METHODOLOGY}

TDDFT is a computationally cheaper method for treating excitonic effects than solving the Bethe-Salpeter equation and gives more accurate results than the tight-binding model (as multi-level and multi-electron effects are included). The evolution of the electronic states during the interaction with twisted light is given by the KS formulation ${ }^{58}$

$$
\begin{aligned}
\mathrm{i} \hbar \frac{\partial}{\partial t}\left|\psi_{i}(r, t)\right\rangle= & {\left[-\frac{\hbar^{2}}{2 m} \nabla^{2}+\nu_{e x t}(r, t)+\nu_{H a}[\rho](r, t)\right.} \\
& \left.+\nu_{x c}[\rho](r, t)\right]\left|\psi_{i}(r, t)\right\rangle \\
\rho(r, t)= & 2 \sum_{i=1}^{M}\left|\psi_{i}(r, t)\right\rangle\left\langle\psi_{i}(r, t)\right|
\end{aligned}
$$

where $\nu_{e x t}, \nu_{H a}$, and $\nu_{x c}$ are the external potential (nuclei and light field), Hartree potential, and exchange-correlation potential, respectively. Eq. (2) is the spin-reduced density operator of $2 M$ electrons. Each time-propagated KS orbital can be expanded in the basis of KS orbitals at $t=0$,

$$
\left|\psi_{i}(r, t)\right\rangle=\sum_{j=1}^{L}\left\langle\psi_{j}(r, 0) \mid \psi_{i}(r, t)\right\rangle\left|\psi_{j}(r, 0)\right\rangle,
$$

with $L$ large enough to make the basis approximately complete. Thus, the multi-electron wave function, which is the Slater determinant of the $M$ occupied time-propagated KS orbitals, can be written as a linear combination of Slater determinants constructed from the $M$ occupied and $L-M$ lowest unoccupied KS orbitals at $t=0$ assuming that all excited states are one-electron excitations ${ }^{59}$,

$$
|\Psi(t)\rangle=c_{0}(t)\left|\psi_{1}(r, 0) \psi_{2}(r, 0) \cdots \psi_{M}(r, 0)\right\rangle+\sum_{i=1}^{M} \sum_{a=1}^{L-M} c_{i}^{a}(t)\left|\Psi_{i}^{a}\right\rangle,
$$

where $\left|\Psi_{i}^{a}\right\rangle=\left|\psi_{1}(r, 0) \cdots \psi_{i}(r, 0) \cdots \psi_{M}(r, 0)\right\rangle$ is the Slater determinant with the $i$-th occupied KS orbital replaced by the $a$-th unoccupied KS orbital.

Real-time TDDFT can be used to generate real KS wave functions at $t=0$. Because of the $C_{N}$ symmetry of the investigated systems, the $\mathrm{KS}$ orbitals with $\mathrm{AM}=0$ are nondegenerate and those with $\mathrm{AM} \neq 0$ are twofold degenerate. Out of each pair of degenerate KS orbitals $\left|\psi_{i}(r, 0)\right\rangle,\left|\psi_{j}(r, 0)\right\rangle$ we can always construct orbitals with opposite AM, specifically $\left|\phi_{i}\right\rangle=$ $\left(\left|\psi_{i}(r, 0)\right\rangle+\mathrm{i}\left|\psi_{j}(r, 0)\right\rangle\right) / \sqrt{2},\left|\phi_{j}\right\rangle=\left(\left|\psi_{i}(r, 0)\right\rangle-\mathrm{i}\left|\psi_{j}(r, 0)\right\rangle\right) / \sqrt{2}$. In Fig. 1 we give examples of this construction for a 3 -arm $\mathrm{H}_{2}$ molecular ring and a triphenylphosphine molecule. First, each real KS orbital is decomposed in the basis of arm orbitals. For example, $\left|\psi_{\mathrm{HOMO}-1}\right\rangle=$ 

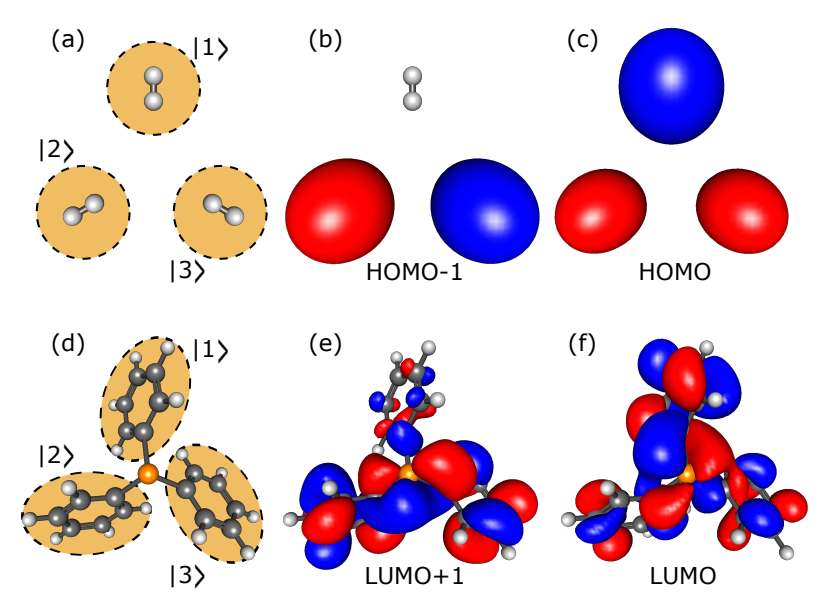

FIG. 1: Arms of (a) a 3-arm $\mathrm{H}_{2}$ molecular ring and (d) a triphenylphosphine molecule. Corresponding wave functions are shown in $(b, c)$ and $(e, f)$, respectively.

$(|2\rangle-|3\rangle) / \sqrt{2},\left|\psi_{\text {номо }}\right\rangle=(-\sqrt{4}|1\rangle+|2\rangle+|3\rangle) / \sqrt{6}$ for the 3 -arm $\mathrm{H}_{2}$ molecular ring and $\left|\psi_{\mathrm{LUMO}+1}\right\rangle=(|2\rangle-|3\rangle) / \sqrt{2},\left|\psi_{\mathrm{LUMO}}\right\rangle=(-\sqrt{4}|1\rangle+|2\rangle+|3\rangle) / \sqrt{6}$ for the triphenylphosphine molecule. For an $N$-arm system the wave function with $\mathrm{AM}=q_{k}$ is

$$
\left|\nu_{q_{k}}\right\rangle=\frac{1}{\sqrt{N}} \sum_{j=1}^{N} \mathrm{e}^{\mathrm{i} 2 \pi(j-1) q_{k} / N}|j\rangle
$$

We then construct

$$
\left|\phi_{k}\right\rangle=\left\langle\psi_{i}(r, 0) \mid \nu_{q_{k}}\right\rangle\left|\psi_{i}(r, 0)\right\rangle+\left\langle\psi_{j}(r, 0) \mid \nu_{q_{k}}\right\rangle\left|\psi_{j}(r, 0)\right\rangle
$$

assuming that $\left|\psi_{i}(r, 0)\right\rangle$ and $\left|\psi_{j}(r, 0)\right\rangle$ are degenerate. This leads to $\left|\phi_{\text {HOMO-1 }}\right\rangle=$ $\left(\left|\psi_{\text {HOмО }-1}\right\rangle+\mathrm{i}\left|\psi_{\text {Hомо }}\right\rangle\right) / \sqrt{2}$ with $\mathrm{AM}=1$ and $\left|\phi_{\text {номо }}\right\rangle=\left(\left|\psi_{\text {HомО }-1}\right\rangle-\mathrm{i}\left|\psi_{\text {HОмо }}\right\rangle\right) / \sqrt{2}$ with $\mathrm{AM}=-1$ for the 3 -arm $\mathrm{H}_{2}$ molecular ring. Similarly, we obtain $\left|\phi_{\mathrm{LUMO}+1}\right\rangle=$ $\left(\left|\psi_{\mathrm{LUMO}+1}\right\rangle+\mathrm{i}\left|\psi_{\mathrm{LUMO}}\right\rangle\right) / \sqrt{2}$ with $\mathrm{AM}=1$ and $\left|\phi_{\mathrm{LUMO}}\right\rangle=\left(\left|\psi_{\mathrm{LUMO}+1}\right\rangle-\mathrm{i}\left|\psi_{\mathrm{LUMO}}\right\rangle\right) / \sqrt{2}$ with $\mathrm{AM}=-1$ for the triphenylphosphine molecule.

Using the new orbitals as basis, the ground state $\left|\phi_{1} \phi_{2} \cdots \phi_{M}\right\rangle$ is identical to $\left|\psi_{1}(r, 0) \psi_{2}(r, 0) \cdots \psi_{M}(r, 0)\right\rangle$, as we have just performed a basis change. For each excited state $\left|\Phi_{k}^{b}\right\rangle$ (Slater determinant for exciting one electron from the $k$-th occupied orbital to the $b$-th unoccupied orbital) the population can be calculated from Eq. (4) as

$$
P_{k}^{b}=\left|\sum_{i=1}^{M} \sum_{a=1}^{L-M}\left\langle\phi_{b} \mid \psi_{a}(r, 0)\right\rangle\left\langle\psi_{i}(r, 0) \mid \phi_{k}\right\rangle c_{i}^{a}(t)\right|^{2} .
$$


As the ground state has $\mathrm{AM}=0$, the nonzero $\mathrm{AM}$ of the excited state $\left|\Phi_{k}^{b}\right\rangle$ must be caused by the replacement of $\left|\phi_{k}\right\rangle$ by $\left|\phi_{b}\right\rangle$. The AM of $\left|\Phi_{k}^{b}\right\rangle$ is therefore obtained by subtracting the AM of $\left|\phi_{k}\right\rangle$ from that of $\left|\phi_{b}\right\rangle$.

While analytically we can calculate by Eq. (7) the population of each excited state and thus the average AM of the exciton $(\overline{\mathrm{XAM}})$, the coefficients of $M$ ! Slater determinants must be obtained for each single population ${ }^{36}$. This is computationally not possible for realistic systems with more than a few electrons. Even for a toy system like a chain of 3 -arm $\mathrm{H}_{2}$ molecular rings we are limited to a maximal length of two rings. However the XAM can be readily extracted from the results of a real-time TDDFT calculation, which allows us to study realistic systems. The AM of $\left|\psi_{i}(r, t)\right\rangle$ is given by

$$
\mathrm{AM}_{i}=\sum_{k=1}^{L}\left|\left\langle\phi_{k} \mid \psi_{i}(r, t)\right\rangle\right|^{2} q_{k},
$$

where $\left|\psi_{i}(r, t)\right\rangle$ is obtained from the TDDFT calculation and $\left|\phi_{k}\right\rangle$ (with $\mathrm{AM}=q_{k}$ ) is calculated by Eqs. (5) and (6). Summation over all time-propagated KS orbitals, $\sum_{i=1}^{M} \mathrm{AM}_{i}$, gives the total AM of the time-propagated multi-electron state, which, however, is a linear combination of ground and excited states. Since the ground state has AM $=0$ and the total number of excitons, $\sum_{j=1}^{L / 2} n_{j}^{2}$, depends only on the positive eigenvalues $\left\{n_{j}\right\}$ of the operator $(\rho(r, t)-\rho(r, 0)) / 2^{60}$, i.e., it is available from the TDDFT calculation, we can readily evaluate the relation

$$
\overline{\mathrm{XAM}}=\sum_{i=1}^{M} \mathrm{AM}_{i} / \sum_{j=1}^{L / 2} n_{j}^{2} .
$$

\section{COMPUTATIONAL RESULTS}

All real-time TDDFT calculations are carried out using OCTOPUS ${ }^{61}$ with TroullierMartins pseudopotentials and the generalized gradient approximation of the exchangecorrelation functional (as parametrized by Perdew, Burke, and Ernzerhof). Spheres of radius $3 \AA$ are chosen around each atom and are discretized by grids with a spacing of $0.15 \AA$. The time step is chosen to be $0.001 \mathrm{fs}$. The Hamiltonian of the light-matter interaction is

$$
\hat{H}_{i n t}(r, t)=-e \vec{r} \cdot \vec{E} \mathrm{e}^{-\left(t-t_{0}\right)^{2} / 2 \tau^{2}} \mathrm{e}^{i \omega t},
$$

where $e$ is the elementary charge and $\vec{E}(|\vec{E}|=0.05 \mathrm{~V} / \AA)$ is the electric field of a circularly polarized light (which is equivalent to twisted light with $\mathrm{AM}= \pm 1^{62}$ ). The light has a 

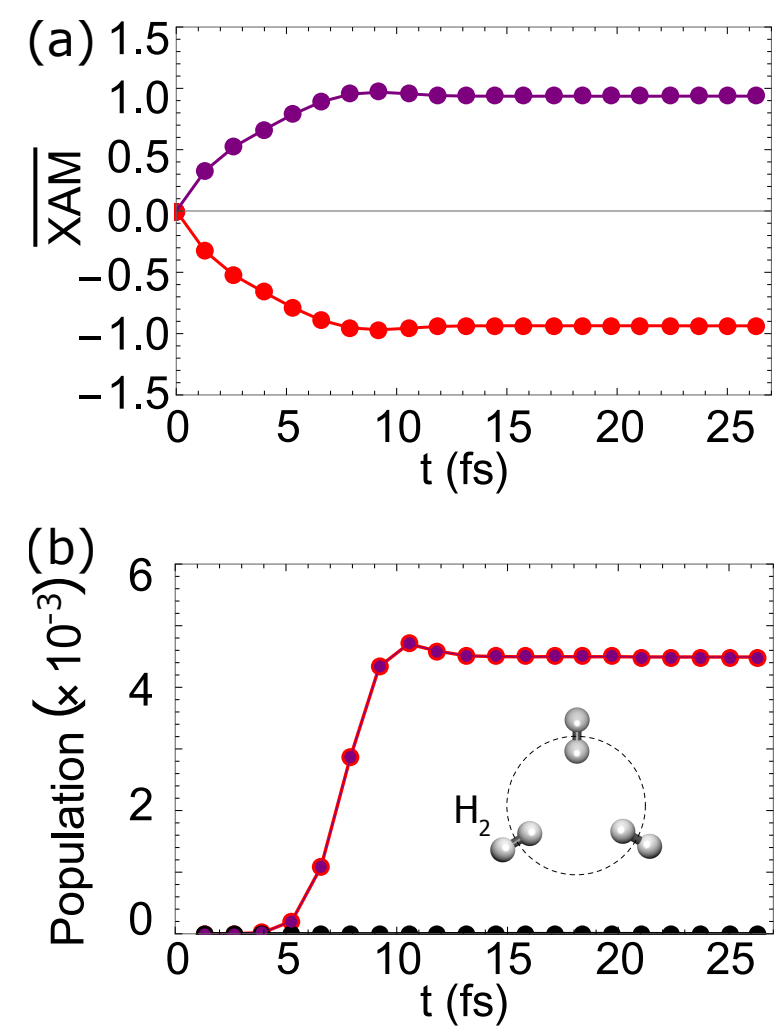

FIG. 2: RT-TDDFT applied to a 3-arm $\mathrm{H}_{2}$ molecular ring illuminated by circularly polarized light with spin 1 (purple) and spin -1 (red): (a) XAM as obtained from Eq. (9). (b) Population of the excited state with $\mathrm{AM}=1$ (purple) and $\mathrm{AM}=-1$ (red). The structure of the 3 -arm $\mathrm{H}_{2}$ molecular ring (radius $2 \AA ; \mathrm{H}-\mathrm{H}$ bond length $0.74 \AA$ ) is shown as inset.

Gaussian envelope with $t_{0}=7.25$ fs and $\tau=1.93$ fs. Its energy $(\hbar \omega)$ is set to $10.6 \mathrm{eV}$ for the systems based on $\mathrm{H}_{2}$ molecular rings and $3.56 \mathrm{eV}$ for the triphenylphosphine molecule.

We first test our methodology by tracking the AM dynamics for a 3-arm $\mathrm{H}_{2}$ molecular ring and a pair of such rings. When a 3-arm $\mathrm{H}_{2}$ molecular ring $(\mathrm{H}-\mathrm{H}$ bond oriented along the radial direction) is illuminated by circularly polarized light with spin \pm 1 propagating perpendicularly to the ring, the generated exciton carries $\overline{\mathrm{XAM}}= \pm 1$ according to Eq. (9), see Fig. 2(a). Eq. (7) implies that all excited states with nonzero population have $\mathrm{AM}= \pm 1$, which is shown in Fig. 2(b). Fig. 3(a) demonstrates for a pair of 3-arm $\mathrm{H}_{2}$ molecular rings with a separation of $20 \AA$ that the exciton also carries $\overline{\mathrm{XAM}}= \pm 1$ when one ring is illuminated by circularly polarized light with spin \pm 1 (possible by two-photon absorption $^{36}$ ). The populations calculated by Eq. (7) are shown in Fig. 3(b). Only excited 

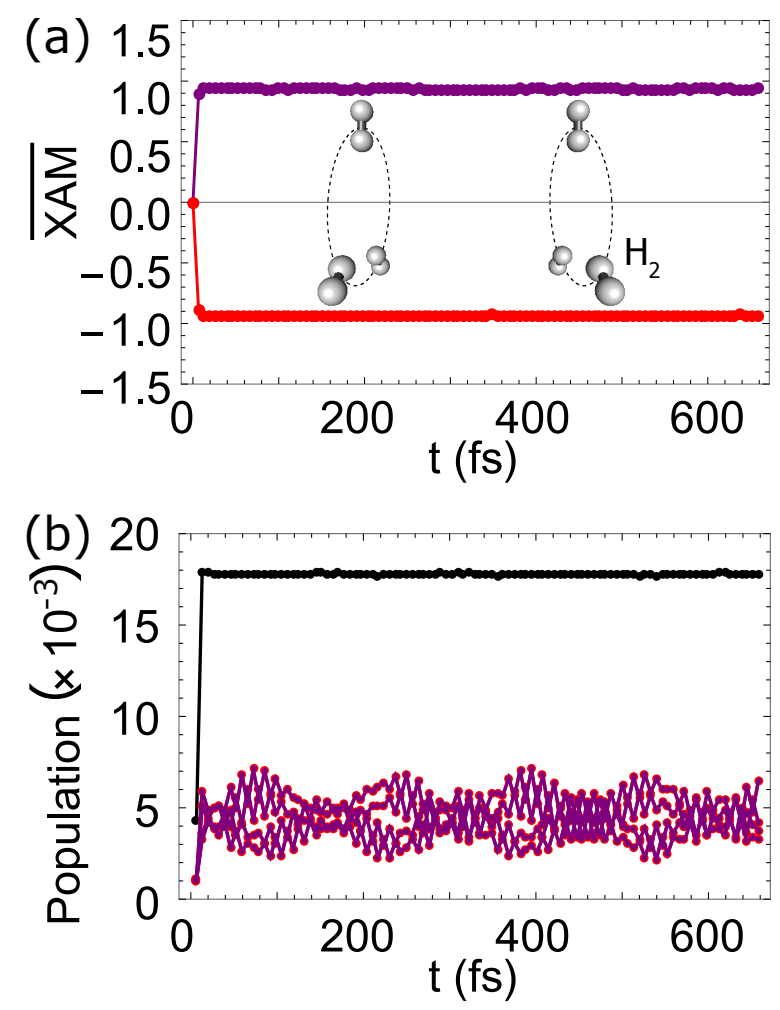

FIG. 3: RT-TDDFT applied to a pair of 3-arm $\mathrm{H}_{2}$ molecular rings with $20 \AA$ separation (see inset) when the first ring is illuminated by circularly polarized light with spin 1 (purple) and spin -1 (red): (a) XAM as obtained from Eq. (9). (b) Populations of the four excited states with AM = 1 (purple) and $\mathrm{AM}=-1$ (red). The total population is shown in black color.

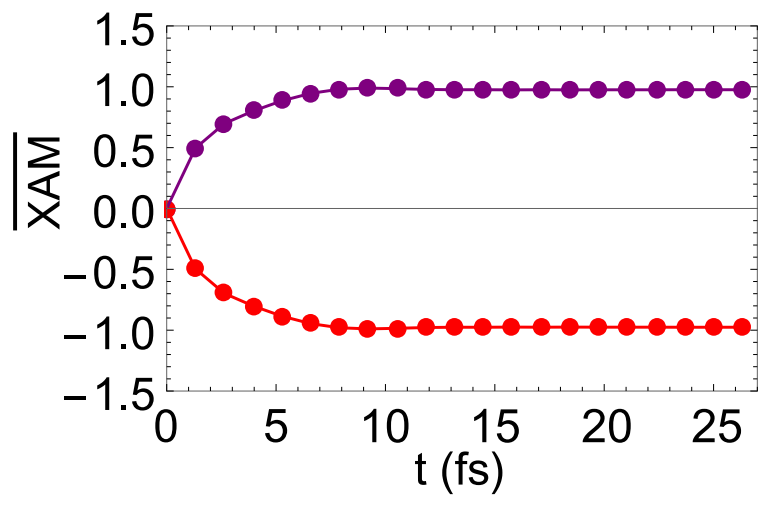

FIG. 4: RT-TDDFT applied to a chain of twenty 3-arm $\mathrm{H}_{2}$ molecular rings with $20 \AA$ separation: $\overline{\mathrm{XAM}}$ as obtained from Eq. (9) when the first ring is illuminated by circularly polarized light with spin 1 (purple) and -1 (red). 
states with $\mathrm{AM}= \pm 1$ have nonzero populations. Summation gives a total population of the exciton (black curve) consistent with the results in Ref. 36.

Having confirmed the reliability of the proposed methodology, we now apply it to a chain of twenty 3-arm $\mathrm{H}_{2}$ molecular rings. This system shows exciton wave packet transfer ${ }^{36}$ but is too complex to derive the $\overline{\mathrm{XAM}}$ analytically. Applying Eq. (9), Fig. 4 confirms that the exciton wave packet carries $\overline{\mathrm{XAM}}= \pm 1$ when the first ring is illuminated by circularly polarized light with spin \pm 1 . Going beyond toy models, we next study a triphenylphosphine molecule. When circularly polarized light with spin \pm 1 is applied along the $C_{3}$ rotational axis, the exciton carries again $\overline{\mathrm{XAM}}= \pm 1$, see Fig. 5(a). Light polarized in a particular direction can be considered as a linear combination of circularly polarized light with spin 1 and spin -1 . When it is applied along the rotational axis of the molecule, states with opposite $\mathrm{AM}$ are excited with equal populations and we have $\overline{\mathrm{XAM}}=0$, see the black
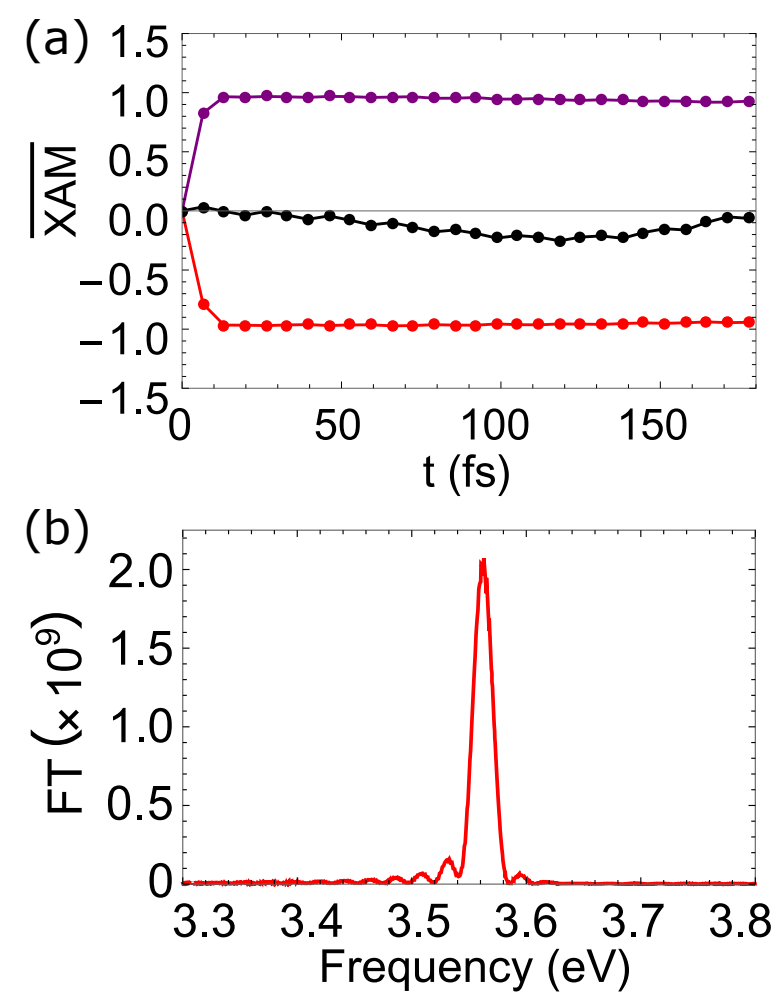

FIG. 5: RT-TDDFT applied to a triphenylphosphine molecule: (a) $\overline{\mathrm{XAM}}$ as obtained from Eq. (9) for illumination by circularly polarized light with spin 1 (purple) and -1 (red) as well as for light polarized perpendicular to the rotational axis of the molecule (black). (b) Fourier transform of the dipole moment. 
(a)

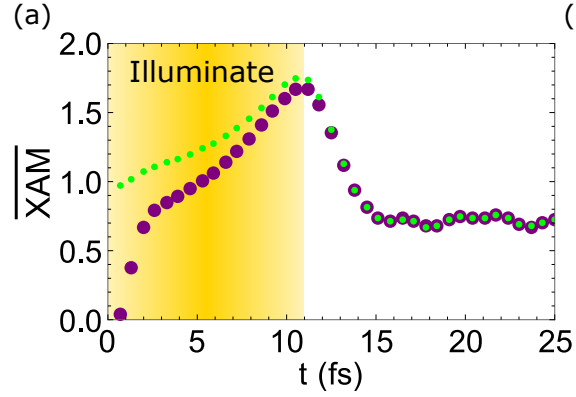

(b) Orbitals

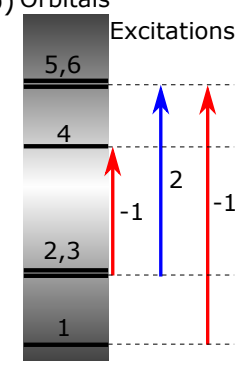

FIG. 6: RT-TDDFT applied to a 3-arm $\mathrm{H}_{2}$ molecular ring illuminated by circularly polarized light with spin -1: (a) XAM calculated by Eq. (9) (purple) compared to analytical results (green). (b) Energy alignment and involved excitations.

curve in Fig. 5(a). The Fourier transform of the dipole moment in Fig. 5(b) shows that the excited states have an energy of $3.56 \mathrm{eV}$. By linear-response TDDFT (OCTOPUS), we find that twofold degenerate states $\left|\Psi_{\mathrm{HOMO}}^{\mathrm{LUMO}}\right\rangle$ and $\left|\Psi_{\mathrm{HOMO}}^{\mathrm{LUMO}+1}\right\rangle$ construct the excited states with $\mathrm{AM}= \pm 1$.

Figure 6(a) shows for a 3-arm $\mathrm{H}_{2}$ molecular ring illuminated by circularly polarized light with spin $-1(\hbar \omega=12 \mathrm{eV})$ that the XAM calculated by Eq. (9) (purple dots) is not -1 . This is caused by mixing of excited states with $\mathrm{AM}=-1$ and 2: Fig. 6(b) depicts the lowest 6 orbitals, with the lowest 3 fully occupied in the ground state. There are 3 dominating excitations, for which our methodology finds $\mathrm{AM}=-1$ or 2 . Comparison to analytical results (green dots), calculated from the populations of the excited states with $\mathrm{AM}=-1$ $\left(P_{-1}\right)$ and $\mathrm{AM}=2\left(P_{2}\right)$ as $\overline{\mathrm{XAM}}=-1 P_{-1}+2 P_{2}$, confirms that the deviation of $\overline{\mathrm{XAM}}$ from a value of -1 is due to the involvement of the excited state with $\mathrm{AM}=2$. Physically, $\mathrm{AM}=2$ is equivalent to $\mathrm{AM}=-1$ because of the reduction of the continuous to a $C_{3}$ rotational symmetry. Similar phenomena have been reported for Wannier-Mott excitons ${ }^{64,65}$.

\section{CONCLUSIONS}

As main result, we have developed a first-principles methodology for extracting the KS AM of an exciton, based on time-dependent density functional theory. In particular, this methodology makes it possible to explore the potential of employing AM as extra degree of freedom in classical and quantum communication. Evaluating the AM of an exciton 
in terms of excited state populations is only possible for small systems. The developed methodology, on the other hand, provides access to realistic systems. The validity of our approach has been confirmed for two toy systems and then applied to a chain of twenty 3-arm $\mathrm{H}_{2}$ molecular rings, for which we have demonstrated that the AM is conserved during the process of illumination by twisted light. For a triphenylphosphine molecule we have shown that the AM again is conserved between twisted light and the created exciton, while the average AM of the exciton is found to be zero when the light is polarized perpendicularly to the rotational axis of the molecule, since in this case the light is a linear combination of circularly polarized light with spin 1 and spin -1 .

\section{ACKNOWLEDGMENTS}

The authors thank Prof. Mark T. Lusk for helpful discussions. The research reported in this publication was supported by funding from King Abdullah University of Science and Technology (KAUST). For computer time, this research used the resources of the Supercomputing Laboratory at KAUST.

* Electronic address: udo.schwingenschlogl@kaust.edu.sa

1 L. Allen, M. W. Beijersbergen, R. J. C. Spreeuw, and J. P. Woerdman, Orbital angular momentum of light and the transformation of Laguerre-Gaussian laser modes, Phys. Rev. A 45, 8185-8189 (1992).

2 G. Gibson, J. Courtial, M. J. Padgett, M. Vasnetsov, V. Pas'ko, S. M. Barnett, and S. FrankeArnold, Free-space information transfer using light beams carrying orbital angular momentum, Opt. Express 12, 5448-5458 (2004).

3 J. Wang, J.-Y. Yang, I. M. Fazal, N. Ahmed, Y. Yan, H. Huang, Y. Ren, Y. Yue, S. Dolinar, M. Tur, and A. E. Willner, Terabit free-space data transmission employing orbital angular momentum multiplexing, Nat. Photon. 6, 488-496 (2012).

4 N. Bozinovic, Y. Yue, Y. Ren, M. Tur, P. Kristensen, H. Huang, A. E. Willner, and S. Ramachandran, Terabit-scale orbital angular momentum mode division multiplexing in fibers, Science 340, 1545-1548 (2013). 
5 A. Mair, A. Vaziri, G. Weihs, and A. Zeilinger, Entanglement of the orbital angular momentum states of photons, Nature 412, 313-316 (2001).

6 A. Vaziri, G. Weihs, and A. Zeilinger, Experimental two-photon, three-dimentional entanglement for quantum communication, Phys. Rev. Lett. 89, 240401 (2002).

7 J. T. Barreiro, N. K. Langford, N. A. Peters, and P. G. Kwiat, Generation of hyperentangled photon pairs, Phys. Rev. Lett. 95, 260501 (2005).

8 E. Nagali, L. Sansoni, F. Sciarrino, F. D. Martini, L. Marrucci, B. Piccirillo, E. Karimi, and E. Santamato, Optimal quantum cloning of orbital angular momentum photon qubits through Hong-Ou-Mandel coalescence, Nat. Photon. 3, 720-723 (2009).

9 J. Leach, B. Jack, J. Romero, A. K. Jha, A. M. Yao, S. Franke-Arnold, D. G. Ireland, R. W. Boyd, S. M. Barnett, and M. J. Padgett, Quantum correlations in optical angular-orbital angular momentum variables, Science 329, 662-665 (2010).

10 J. Leach, E. Bolduc, D. J. Gauthier, and R. W. Boyd, Secure information capacity of photons entangled in many dimensions, Phys. Rev. A 85, 060304 (2012).

11 M.Mafu, A. Dudley, S. Goyal, D. Giovannini, M. McLaren, M. J. Padgett, T. Konrad, F. Petruccione, N. Lütkenhaus, and A. Forbes, Higher-dimensional orbital-angular-momentumbased quantum key distribution with mutually unbiased bases, Phys. Rev. A 88, 032305 (2013).

12 G. Vallone, V. D'Ambrosio, A. Sponselli, S. Slussarenko, L. Marrucci, F. Sciarrino, and P. Villoresi, Free-space quantum key distribution by rotation-invariant twisted photons, Phys. Rev. Lett. 113, 060503 (2014).

13 M. McLaren, T. Mhlanga, M. J. Padgett, F. S. Roux, and A. Forbes, Self-healing of quantum entanglement after an obstruction, Nat. Commun. 5, 3248 (2014).

14 M. Mirhosseini, O. S. Magaña-Loaiza, M. N. O’Sullivan, B. Rodenburg, M. Malik, M. P. J. Lavery, M. J. Padgett, D. J. Gauthier, and R. W. Boyd, High-dimensional quantum cryptography with twisted light, New J. Phys. 17, 033033 (2015).

15 V. D'Ambrosio, G. Carvacho, F. Graffitti, C. Vitelli, B. Piccirillo, L. Marrucci, and F. Sciarrino, Entangled vector vortex beams, Phys. Rev. A 94, 030304 (2016).

16 N. R. Heckenberg, R. McDuff, C. P. Smith, and A. G. White, Generation of optical phase singularities by computer-generated holograms, Opt. Lett. 17, 221-223 (1992).

17 A. S. Ostrovsky, C. Rickenstorff-Parrao, and V. Arrizón, Generation of the "perfect" optical vortex using a liquid-crystal spatial light modulator, Opt. Lett. 38, 534-536 (2013). 
18 M. Beijersbergen, R. Coerwinkel, M. Kristensen, and J. Woerdman, Helical-wavefront laser beams produced with a spiral phaseplate, Opt. Commun. 112, 321-327 (1994).

19 L. Marrucci, C. Manzo, and D. Paparo, Optical spin-to-orbital angular momentum conversion in inhomogeneous anisotropic media, Phys. Rev. Lett. 96, 163905 (2006).

20 E. Nagali, F. Sciarrino, F. De Martini, L. Marrucci, B. Piccirillo, E. Karimi, and E. Santamato, Quantum information transfer from spin to orbital angular momentum of photons, Phys. Rev. Lett. 103, 013601 (2009).

21 E. Brasselet, N. Murazawa, H. Misawa, and S. Juodkazis, Optical vortices from liquid crystal droplets, Phys. Rev. Lett. 103, 103903 (2009).

22 L. Marrucci, E. Karimi, S. Slussarenko, B. Piccirillo, E. Santamato, E. Nagali, and F. Sciarrino, Spin-to-orbital conversion of the angular momentum of light and its classical and quantum applications, J. Opt. 13, 064001 (2011).

23 A. Ciattoni, G. Cincotti, and C. Palma, Circularly polarized beams and vortex generation in uniaxial media, J. Opt. Soc. Am. A 20, 163-171 (2003).

24 A. Ciattoni, G. Cincotti, and C. Palma, Angular momentum dynamics of a paraxial beam in a uniaxial crystal, Phys. Rev. E 67, 036618 (2003).

25 E. Brasselet, Y. Izdebskaya, V. Shvedov, A. S. Desyatnikov, W. Krolikowski, and Y. S. Kivshar, Dynamics of optical spin-orbit coupling in uniaxial crystals, Opt. Lett. 34, 1021-1023 (2009).

26 C. Loussert and E. Brasselet, Efficient scalar and vectorial singular beam shaping using homogeneous anisotropic media, Opt. Lett. 35, 7-9 (2010).

27 T. A. Fadeyeva, V. G. Shvedov, Y. V. Izdebskaya, A. V. Volyar, E. Brasselet, D. N. Neshev, A. S. Desyatnikov, W. Krolikowski, and Y. S. Kivshar, Spatially engineered polarization states and optical vortices in uniaxial crystals, Opt. Express 18, 10848-10863 (2010).

28 M. D. Williams, M. M. Coles, K. Saadi, D. S. Bradshaw, and D. L. Andrews, Optical vortex generation from molecular chromophore arrays, Phys. Rev. Lett. 111, 153603 (2013).

29 M. D. Williams, M. M. Coles, D. S. Bradshaw, and D. L. Andrews, Direct generation of optical vortices, Phys. Rev. A 89, 033837 (2014).

30 F. Bouchard, I. D. Leon, S. A. Schulz, J. Upham, E. Karimi, and R. W. Boyd, Optical spinto-orbital angular momentum conversion in ultra-thin metasurfaces with arbitrary topological charges, Appl. Phys. Lett. 105, 101905 (2014).

31 J. Jin, J. Luo, X. Zhang, H. Gao, X. Li, M. Pu, P. Gao, Z. Zhao, and X. Luo, Generation and 
detection of orbital angular momentum via metasurface, Sci. Rep. 6, 24286 (2016).

J. Zeng, L. Li, X. Yang, and J. Gao, Generating and separating twisted light by gradient-rotation split-ring antenna metasurfaces, Nano Lett. 16, 3101-3108 (2016).

33 M. Krenn, J. Handsteiner, M. Fink, R. Fickler, R. Ursin, M. Malik, and A. Zeilinger, Twisted light transmission over 143 km, Proc. Natl. Acad. Sci. 113, 13648-13653 (2016).

${ }^{34}$ R. Fickler, G. Campbell, B. Buchler, P. K. Lam, and A. Zeilinger, Quantum entanglement of angular momentum states with quantum numbers up to 10,010, Proc. Natl. Acad. Sci. 113, 13642-13647 (2016).

35 X. Zang and M. T. Lusk, Twisted molecular excitons as mediators for changing the angular momentum of light, Phys. Rev. A 96, 013819 (2017).

36 X. Zang and M. T. Lusk, Angular momentum transport with twisted exciton wave packets, Phys. Rev. B 96, 155104 (2017).

37 N. H. Bonadeo, J. Erland, D. Gammon, D. Park, D. S. Katzer, and D. G. Steel, Coherent optical control of the quantum state of a single quantum dot, Science 282, 1473-1476 (1998).

38 T. H. Stievater, Xiaoqin Li, D. G. Steel, D. Gammon, D. S. Katzer, D. Park, C. Piermarocchi, and L. J. Sham, Rabi oscillations of excitons in single quantum dots, Phys. Rev. Lett. 87, $133603(2001)$.

39 A. Zrenner, E. Beham, S. Stufler, F. Findeis, M. Bichler, and G. Abstreiter, Coherent properties of a two-level system based on a quantum-dot photo-diode, Nature 418, 612-614 (2002).

40 H. Htoon, T. Takagahara, D. Kulik, O. Baklenov, A. L. Holmes, and C. K. Shih, Interplay of Rabi oscillations and quantum interference in semiconductor quantum dots, Phys. Rev. Lett. 88, 087401 (2002).

41 L. Besombes, J. J. Baumberg, and J. Motohisa, Coherent spectroscopy of optically gated charged single ingaas quantum dots, Phys. Rev. Lett. 90, 257402 (2003).

42 B. Patton, U. Woggon, and W. Langbein, Coherent control and polarization readout of individual excitonic states, Phys. Rev. Lett. 95, 266401 (2005).

43 R. Hildner, D. Brinks, and N. F. van Hulst, Femtosecond coherence and quantum control of single molecules at room temperature, Nat. Phys. 7, 172-177 (2011).

44 D. Brinks, R. Hildner, E. M. H. P. van Dijk, F. D. Stefani, J. B. Nieder, J. Hernando, and N. F. van Hulst, Ultrafast dynamics of single molecules, Chem. Soc. Rev. 43, 2476-2491 (2014).

45 T. Lundstrom,W. Schoenfeld, H. Lee, and P. M. Petroff, Exciton storage in semiconductor 
self-assembled quantum dots, Science 286, 2312-2314 (1999).

46 A. G. Winbow, A. T. Hammack, L. V. Butov, and A. C. Gossard, Photon storage with nanosecond switching in coupled quantum well nanostructures, Nano Lett. 7, 1349-1351 (2007).

47 M. Hagn, A. Zrenner, G. Böhm, and G. Weimann, Electric-field-induced exciton transport in coupled quantum well structures, Solid-State Electron. 40, 429-431 (1996).

48 A. Gartner, A. W. Holleitner, J. P. Kotthaus, and D. Schuh, Drift mobility of long-living excitons in coupled GaAs quantum wells, Appl. Phys. Lett. 89, 052108 (2006).

49 M. T. Lusk, C. A. Stafford, J. D. Zimmerman, and L. D. Carr, Control of exciton transport using quantum interference, Phys. Rev. B 92, 241112 (2015).

50 A. A. High, A. T. Hammack, L. V. Butov, M. Hanson, and A. C. Gossard, Exciton optoelectronic transistor, Opt. Lett. 32, 2466-2468 (2007).

51 A. A. High, E. E. Novitskaya, L. V. Butov, M. Hanson, and A. C. Gossard, Control of exciton fluxes in an excitonic integrated circuit, Science 321, 229-231 (2008).

52 R. J. Warburton, Optoelectronics: Electronics lightens up, Nat. Phys. 4, 676-677 (2008).

53 A. G. Winbow, J. R. Leonard, M. Remeika, Y. Y. Kuznetsova, A. A. High, A. T. Hammack, L. V. Butov, J. Wilkes, A. A. Guenther, A. L. Ivanov, M. Hanson, and A. C. Gossard, Electrostatic conveyer for excitons, Phys. Rev. Lett. 106, 196806 (2011).

54 A. Perdomo, L. Vogt, A. Najmaie, and A. Aspuru-Guzik, Engineering directed excitonic energy transfer, Appl. Phys. Lett. 96, 093114 (2010).

55 S. M. Menke, T. K. Mullenbach, and R. J. Holmes, Directing energy transport in organic photovoltaic cells using interfacial exciton gates, ACS Nano 9, 4543-4552 (2015).

56 D. Unuchek, A. Ciarrocchi, A. Avsar, K. Watanabe, T. Taniguchi, and A. Kis, Roomtemperature electrical control of exciton flux in a van der Waals heterostructure, Nature $\mathbf{5 6 0}$, 340-344 (2018).

57 X. Zang, S. Montangero, L. D. Carr, and M. T. Lusk, Engineering and manipulating exciton wave packets, Phys. Rev. B 95, 195423 (2017).

58 E. Runge and E. K. U. Gross, Density-functional theory for time-dependent systems, Phys. Rev. Lett. 52, 997-1000 (1984).

59 C. F. Craig, W. R. Duncan, and O. V. Prezhdo, Trajectory surface hopping in the timedependent Kohn-Sham approach for electron-nuclear dynamics, Phys. Rev. Lett. 95, 163001 $(2005)$. 
60 M. Head-Gordon, A. M. Grana, D. Maurice, and C. A. White, Analysis of electronic transitions as the difference of electron attachment and detachment densities, J. Phys. Chem. 99, 1426114270 (1995).

61 X. Andrade, D. Strubbe, U. De Giovannini, A. H. Larsen, M. J. T. Oliveira, J. AlberdiRodriguez, A. Varas, I. Theophilou, N. Helbig, M. J. Verstraete, L. Stella, F. Nogueira, A. Aspuru-Guzik, A. Castro, M. A. L. Marques, and A. Rubio, Real-space grids and the Octopus code as tools for the development of new simulation approaches for electronic systems, Phys. Chem. Chem. Phys. 17, 31371-31396 (2015).

62 Q. Zhan, Properties of circularly polarized vortex beams, Opt. Lett. 31, 867-869 (2006).

63 M. Damnjanović and I. Milošević, Line groups in physics (Springer, Boston, 2010).

64 J. Thewes, J. Heckötter, T. Kazimierczuk, M. Aßmann, D. Fröhlich, M. Bayer, M. A. Semina, and M. M. Glazov, Observation of high angular momentum excitons in cuprous oxide, Phys. Rev. Lett. 115, 027402 (2015).

65 M. M. Glazov, L. E. Golub, G. Wang, X. Marie, T. Amand, and B. Urbaszek, Intrinsic excitonstate mixing and nonlinear optical properties in transition metal dichalcogenide monolayers, Phys. Rev. B 95, 035311 (2017). 